\title{
A CERIF Compatible CRIS-UNS Model Extension for Assessment of Conference Papers
}

\author{
Siniša Nikolić*, Zora Konjović*, Valentin Penca*, Dragan \\ Ivanović*, Dušan Surla** \\ *University of Novi Sad, Faculty of Technical Sciences \\ Trg Dositeja Obradovića 6, 21000 Novi Sad, Serbia, sinisa_nikolic@uns.ac.rs, \\ ftn_zora@uns.ac.rs, valentin_penca@uns.ac.rs, chenejac@uns.ac.rs \\ **University of Novi Sad, Faculty of Sciences \\ Trg Dositeja Obradovića 3, 21000 Novi Sad, Serbia, surla@uns.ac.rs
}

\begin{abstract}
This paper proposes an extension to CERIF compatible CRIS, enabling automated evaluation of research achievements by applying diverse (country/region/institution specific), or even multiple evaluation rulebooks and guidelines. It was implemented as an extension to the CERIF compliant CRIS system of the University of Novi Sad (CRIS UNS) that already contains information for assessment of results from scientific journals, so the focus of this research is an extension to the CERIF model aimed at evaluation of the results published through conferences. Based on a survey and an analysis of selected evaluation rulebooks and guidelines, the paper proposes an extended CERIF model that comprises conference evaluation related metadata and a machinereadable representation of a rulebook that enables automated evaluation. A rule-based expert system is proposed for representation of evaluation rules and evaluation of research results. The Serbian rulebook is represented and implemented using the expert system Jess in order to evaluate the proposed model. Reliance of the model on CERIF standard allows its easy application in any CERIF compatible CRIS system.
\end{abstract}

Keywords: CERIF; model extension; conferences; automated evaluation; Jess

\section{Introduction}

In recent years, as research and innovations are becoming crucially important for economic development and Government financing is tightening, assessment of research achievements and capacities become an unavoidable condition for identifying high quality research, both for strategic planning and other purposes, like appointments to scientific/teaching positions, ranking researchers and/or research institutions, decisions on scientific project financing, etc. [1]. The Committee for Evaluation of Research defines research evaluation as a process based on critical analysis of data which leads to a judgment of merit [2]. Research 
outputs, such as monographs/books, journal articles and conference papers are the evidence of a research study findings and they are the most suitable for assessment. Evaluation of conference proceedings papers is not as extensive as the evaluation of journal articles. This could be explained by the well-established opinion that journals present scientific results of the highest quality, but that cannot be an excuse, as conference papers have a capacity to exceed journal papers regarding the up-to-date presentation of ideas. This is due to a relatively short review time for scientific conferences. Also, for some fast-growing scientific areas (e.g. Computer Science - CS), conference papers are the major form of publishing (conference-cantered publication culture) [3]. As the papers published at conferences are a significant part of scientific production, it is necessary to investigate the assessment of those research results as well.

Electronic databases containing research outputs were, and still are, a basis for research evaluation process. Construction of an information system is necessary for efficient evaluation of scientific-research data [4]. The Current Research Information System (CRIS) that is based on the Common European Research Information Format (CERIF) standard is an example of such a system. It represents a good base for development of a system for evaluation of scientific results.

Usually, the evaluation process is carried out by a commission of domain experts that decide on huge amounts of publications by following some evaluation rulebook or set of guidelines that could be subject to change and/or subjective interpretation. Therefore, the evaluation should be supported by an evaluation engine that will apply rules automatically, provide explanations of the evaluation process, and even support an option for rewriting rules, if necessary. An expert system in which evaluation rules are expressed by some declarative language could be a solution to the problem.

\section{Related Work}

The fundamental concepts underlying the research presented in this paper represent an approach to evaluation (objects of evaluation, procedures and metrics applied to evaluate objects, entities that carry out evaluation) and information resources and software tools that are used to assist evaluation.

In practice, research performance evaluation, mainly relies on the quality of the resulting publications, i.e. scientific publications are primary objects of evaluation. Other objects of evaluation (e.g. researchers, research institutions, etc.) are evaluated mainly relying upon evaluations of the corresponding primary objects. As stated in [5], the quality of a publication can be determined by using different approaches: expert opinion (peer review), bibliometric evaluation, or a combination of these two (experts that use bibliometric data for their decisions, 
the most acceptable approach to evaluation of research results so far). The authors of the paper [6] state that rankings have become one of the main forms of quality assessment in higher education over the past few decades. Publication rankings are based on individual (each publication gains individual score) or collective (all publications gain the same score as a part of a larger publication, with an optional coarse granulation like scientific paper, professional paper) evaluation of publications. Many evaluation principles are based on collective evaluation, arguing that assessment of the source (e.g. journal, conference) in which the article is published is unbiased, less time- and resource-consuming, and more economical than the individual publication evaluation [7]. So, the "quality/reputation" of a conference can be a dominant criterion when assessing the quality of conference results.

One of the most successful attempts to rank and evaluate conferences, so far, is a subjective CORE ranking [8]. Examples of conference rankings that are based on a voting procedure (classification) are ERA $2010^{1}$ and Perfil-CC ${ }^{2}$ Ranking for computer science area. The rankings mentioned above indicate that expert evaluation is only carried out for a relatively small number of conferences, usually for a particular scientific field and to satisfy the need of some country or geographical area. Evaluation on a larger scale requires use of some metric for conferences like acceptance rates, bibliometric indicators, and bibliometric related data [9].

Once decided on the assessment approach, it is necessary to have access to data required to carry out evaluation. Regarding this issue, it is important to mention that there are scientific publication databases, which store some metrics that can be used to evaluate conferences. Some of those databases are Google Scholar Metrics $^{3}$ (GSM), Microsoft Academic ${ }^{4}$ (MA), Web of Science Conference Proceedings Citation Index ${ }^{5}$ (CPCI), Elsevier Scopus ${ }^{6}(E S)$ and CiteSeer Estimated Venue Impact Factors ${ }^{7}(E V I F)$. GSM, MA, ES and CPCI contain bibliography and citation data for proceedings articles. MA provides ranking of conferences, while GSM and EVIF provide single list ranking of journals and conferences, meaning that for ranking of conferences only journals must be somehow excluded. GSM, MA, and EVIF have free access; while ES and CPCI are commercially available products (access is for commercial subscribers only). CPCI contains the highest amount of data compared to other databases and can be indirectly used to create conference evaluation metrics. Considering domain

\footnotetext{
${ }^{1} \mathrm{http} / / /$ lamp.infosys.deakin.edu.au/era/?page $=$ cforsel10

$2 \mathrm{http} / / /$ www.latin.dcc.ufmg.br/perfilccranking/

${ }^{3} \mathrm{https}: / /$ scholar.google.com/citations?view_op=top_venues\&hl=en

${ }^{4} \mathrm{http}: / /$ academic.research.microsoft.com/

${ }_{6}^{5} \mathrm{http} / / /$ thomsonreuters.com/conference-proceedings-citation-index/

${ }^{6} \mathrm{http}: / /$ www.scopus.com/

${ }^{7}$ http://citeseerx.ist.psu.edu/stats/venues
} 
coverage and usability, these databases are severely limited in research scope and data completeness. They cover mostly the area of Computer Science and sometimes Electrical Engineering, and even for those, the data is not as complete as for journal articles.

In addition to the abovementioned data sources, there are databases and repositories of research institutions and/or states which are considerable and valuable information resources for conference results evaluation. Numerous institutional/state databases/repositories provide data in accordance with CERIF model. CERIF is an open [10], widespread [11] international standard, with an already proven capacity for research results evaluation [12, 13] and clearly recognized by European Science Foundation for that purpose [14]. An important advantage of CERIF is that it can be extended and adapted to different needs. This has already been proven through results, such as those aimed at storing bibliometric indicators [15], and an extension aimed at evaluation of journal papers in CRIS UNS, presented in [16]. The latest activities, aimed at CERIF integration, with complementing standards aimed to support effective evidencebased institutional decision-making are in progress [17]. All this makes CERIF an important reference point for the purpose of evaluation regulated by national rulebooks.

In order to enable efficient evaluation, it is necessary to provide evaluators with tools that will automate evaluation process as much as possible. In an evaluation of research results, where rules from rulebooks are applied by some commissions, an obvious approach to evaluation automation is to use some rule-based expert system. Unlike the conventional systems that solve a problem by executing a welldefined algorithm, expert systems rely upon a knowledge base that contains statements and facts about the problem [18]. The advantage of a rule-based (declarative programming) code over a conventional programming code is that it is much easier to read, maintain and change, even by those who are not familiar with programming [19].

\section{Analysis of National Rulebooks and Guidelines}

The purpose of this section is to analyze evaluations at the national levels that apply to papers that are presented at conferences. In general, evaluations at national levels differ from one country to another. For this analysis, we have chosen representative countries differing in size, economic development, geolocation, and relation to the EU. In the rest of this section, country specific rulebooks and/or guidelines are analyzed for four non EU - Southern European countries (Serbia, Macedonia, Montenegro, Bosnia and Herzegovina), five EU countries (Croatia, Slovenia, Czech Republic, Hungary, United Kingdom), and one non-European country (Australia). 
The evaluation of research results in Serbia utilizes the "combined" approach. For evaluation purposes, commissions use the document that was prescribed by the Serbian Ministry of Education and Science ${ }^{8}$. The results presented at conferences are evaluated based on the category of a conference and type of presentation and result. Conferences are categorized as international or national by the commission that is in charge of a scientific field corresponding to the scope of a conference.

The rulebook of Bosnia and Herzegovina is identical to the Serbian rulebook in terms of data requirements ${ }^{9}$. It prescribes the same categorization as the Serbian rulebook, differing only in categorization codes.

In Macedonia, the assessment of researchers' results is done by a commission. The commission uses the document that is created by the leading Macedonian university - University Ss. Cyril and Methodius in Skopje ${ }^{10}$. Conference results are categorized on the basis of conference evaluation. There are two types of conferences: a scientific/expert conference with an international program committee and a scientific/expert conference. Conferences that have an international committee with members from at least 5 countries, where the number of members from the most represented country does not exceed $40 \%$, are ranked as higher quality conferences.

In Montenegro, the evaluation approach is almost the same as in Serbia and Macedonia (done by a commission and relying on bibliometric data). A sort of specific quality of the Montenegrin rulebook ${ }^{11}$ is that the information on editors is used for conference proceedings results evaluation. Conference results are categorized on the basis of evaluation of a conference. The evaluation of a conference relies on: proceedings publication language (worldwide accepted languages are favored), proceedings editorial committee structure (international committees with distinguished members are favored) and organizer (international organizers are favored).

The Croatian evaluation rulebook ${ }^{12}$ and its rules for classifying conference results are based on the evaluation of a conference. The conference category is determined based on the organizer (organizer must be a part of a specially verified list - organizer type) and the indexing of conference proceeding in CPCI (proceeding must be a part of list). A conference is categorized as international if organized by an international scientific association or if its proceeding is indexed in CPCI.

\footnotetext{
${ }^{8}$ http://www.mpn.gov.rs/images/content/nauka/pravna_akta/PRAVILNIK_o_zvanjima.pdf

${ }^{9} \mathrm{http}: / /$ mcp.gov.ba/org_jedinice/sektor_nauka_kultura/pravni_okvir/podzakonski_akti/defa ult.aspx?id=3379\&langTag=bs-BA

${ }^{10} \mathrm{http} / / /$ www.ffk.pesh.mk/Vazni_dokumenti/Pravni_dokumenti/22_133786659.pdf

${ }^{11} \mathrm{http} / / /$ www.ucg.ac.me/zakti/akademska_zvanja.pdf

${ }^{12} \mathrm{http}: / /$ narodne-novine.nn.hr/clanci/sluzbeni/2013_03_26_447.html
} 
The Slovenian rulebook ${ }^{13}$ assesses researchers' results based on a set of criteria for research excellence (evaluation of researchers' publications and citations). Scientific contributions from conferences are categorized by the evaluation of a conference. By the Slovenian rulebook, conferences that take place abroad or those organized in Slovenia in worldwide accepted languages, with an international committee and a minimum of one-third of publications from abroad gain the highest score. The particulars of the Slovenian rulebook are two predefined time frames for citation (last 5 and 10 years). The total number of citations and the number of pure citations (citations without auto-citations) in the CPCI for the defined time frames are required for conference papers.

The Czech rulebook ${ }^{14}$ evaluates each submitted publication by using bibliometric data or scientific area experts within panels. Publications from conferences are not preferable in some scientific areas (not accepted, or included in a share of 50\%, $25 \%$, or below $5 \%$ of all publications). Conference papers are evaluated directly by experts in panels (that approach does not require evaluation of the conference itself). A conference proceedings paper is eligible for evaluation only if over 2 pages in length and if the conference proceeding in which the paper is published is indexed in CPCI or Scopus (proceeding must be a part of CPCI or Scopus list).

$\mathrm{PhD}$ and Habilitation committees at the Faculty of Science and Information Technology in Szeged, Hungary, directly evaluate conference papers. The Document ${ }^{15}$ is used as a guideline for that evaluation. What is particular for this evaluation compared to the others is that it accounts for pure citations which are not derived from the author's affiliation, pure citations from any source and pure citations that are only found in a predefined list of databases (e.g. Web of Science and Scopus). For a conference paper to be considered for evaluation, its conference proceeding must be indexed in a predefined list of databases (e.g. Mathematical Reviews, WoS, CPCI, Scopus etc.) or its conference must be an internationally recognized event (symposium and workshop) with an acceptance rate below $50 \%$ and the ratio of international authors above $50 \%$. According to commissions, a conference is international if the participants and the committee are international. Since those conditions are not formally supported with some metrics, such as the numbers of authors and committee members (no such details are used, as Committee members structure and Results data are used in other rulebooks), there are no formal criteria for representing them. The quality of each conference paper is determined based on the opinion of commissions, categorizing them on a 3 grade scale as International in English, Domestic in English or In Hungarian.

\footnotetext{
${ }^{13} \mathrm{http}: / /$ www.arrs.gov.si/en/akti/prav-sof-ocen-sprem-razisk-dej-sept-11.asp

${ }^{14} \mathrm{http}: / / \mathrm{www}$. vyzkum.cz/FrontClanek.aspx ?idsekce=695512\&ad=1\&attid=695694

${ }^{15} \mathrm{http} / / /$ www.sci.u-szeged.hu/kar/kari-szabalyzatok/ttik-doktori-szabalyzat? objectParentFolderId=19613
} 
The United Kingdom has created an assessment framework called $\mathrm{REF}^{16}$ (Research Excellence Framework). According to REF, conference papers are directly evaluated by experts on Units of Assessments (panels). A particular quality of REF is that it requires data about the abstract of publication. In some cases, citation data might be utilized in evaluation (for a particular scientific area, if it is available and if experts would like to use it). REF states that all research output data requirements are compatible with CERIF.

ERA $^{17}$ (Excellence in Research for Australia) assessment framework in Australia is very close to REF, i.e. conference papers are evaluated by experts in panels. Some panels accept peer review evaluation, but only for up to $30 \%$ of the submitted publications, so conference proceedings papers are only evaluated by this approach.

\subsection{Metadata for Evaluation of Conference Papers}

The analysis of rulebooks and guidelines leads to the conclusion that all national rulebooks somehow include conference papers. In general, scores are assigned to conference proceeding articles either based on the opinion of the experts in the panel (experts' judgment which can, up to a certain extent, rely upon bibliometric indicators), or by a commission applying the rules, relying upon conference categories, where a conference category is determined based on common criteria (language, structure of a scientific committee, etc.), and, sometimes, on bibliometric indicators that apply to conference proceedings (CPCI indexing). In some rulebooks, when assessing publications presented at conferences, in addition to the metadata describing the publication itself, it is necessary to include the data for the conference and conference proceedings. Therefore, metadata consists of three sets: conference metadata, proceedings metadata and publication metadata.

As a result of the rulebooks' analysis, the following is a set of evaluation metadata for conferences (Table 1) that all rulebooks include: conference name, year, place, presentation language, proceeding data, conference committee structure, organizer data and conference results data. In most of the rulebooks, the category of a conference depends on the conference organizer, conference language, proceeding data and conference committee structure. The conference committee structure metadata includes the total number of committee members, total number of countries from which committee members originate, number of committee members from the most represented country. The metadata on the conference organizer describe the particular organizer (name of institution) and organizer type (international, national). The conference results data is described with the total

\footnotetext{
${ }^{16} \mathrm{http}: / /$ www.ref.ac.uk/media/ref/content/pub/panelcriteriaandworkingmethods/01_12.pdf

${ }^{17} \mathrm{http} / / /$ www.arc.gov.au/pdf/era12/ERA\%202012\%20Evaluation\%20Handbook_final\%20f or\%20web_protected.pdf
} 
number of papers, total number of submitted papers and number of papers whose authors are foreigners.

Table 1

Evaluation metadata for conferences

\begin{tabular}{|c|c|c|c|c|c|c|c|c|c|c|}
\hline & Serbia & Macedonia & Montenegro & $\begin{array}{c}\text { Bosnia and } \\
\text { Herregovina }\end{array}$ & Croatia & Slovenia & $\begin{array}{c}\text { Czech } \\
\text { Republick }\end{array}$ & Hungary & $\begin{array}{c}\text { United } \\
\text { Kingdom }\end{array}$ & Australia \\
\hline name & + & + & + & + & + & + & + & + & + & + \\
\hline year & + & + & + & + & + & + & + & & & + \\
\hline place & + & + & + & + & + & + & + & & & + \\
\hline organizer & + & & + & + & + & & & & & \\
\hline presentation language & + & & & + & & + & & & & \\
\hline proceeding & + & + & + & + & & & & & & \\
\hline $\begin{array}{l}\text { conference committee structure: total number of } \\
\text { committee members }\end{array}$ & + & + & & + & & + & & & & \\
\hline $\begin{array}{l}\text { conference committee structure: total number of } \\
\text { countries from which the committee members } \\
\text { originate }\end{array}$ & + & + & & + & & + & & & & \\
\hline $\begin{array}{l}\text { conference committee structure: number of committee } \\
\text { members from the most represented country }\end{array}$ & & + & & & & & & & & \\
\hline organizer data: organizer type & + & & + & + & + & & & & & \\
\hline conference results data: total number of papers & + & & & + & & + & & + & & \\
\hline conference results data: number of submitted papers & & & & & & & & + & & \\
\hline $\begin{array}{l}\text { conference results data: number of papers whose } \\
\text { authors are foreigners }\end{array}$ & + & & & + & & + & & + & & \\
\hline
\end{tabular}

Conference proceeding (Table 2) may include data as proceedings: title, ISBN, publication language, publisher, editorial committee structure and indexing of the conference proceeding in CPCI and/or SCOPUS. Publication language, indexing and editorial committee structure are used to categorize a conference. The editorial committee data, which is used only by the Montenegro rulebook, consists of name(s) of editor(s), total number of editorial committee members and total number of countries from which the committee members originate. Indexing of conference proceedings in CPCI and/or SCOPUS is required only for the Croatian and Czech Republic rulebooks. In Hungary, it is preferred that the conference proceeding be indexed in a predefined list of databases. The metadata describing the publisher are publisher's name and headquarters of the publisher.

A conference publication (Table 3) is defined by the following data: title, author(s) name(s), publication year, total number of pages, conference data, proceedings data, citation data, DOI, URL, scientific area, abstract and type of evaluation entity. The citation data is required only in the UK (total number of citations), Slovenia (total number of citations, number of pure citations in the last 5 and number of pure citations in the last 10 years) and Hungary (total number of citations, number of pure citations, number of pure citations not derived from the author's own affiliation, number of pure citations found in a predefined list of databases). 
Table 2

Evaluation metadata for conference proceedings

\begin{tabular}{|c|c|c|c|c|c|c|c|c|c|c|}
\hline & Serbia & Macedonia & Montenegro & $\begin{array}{l}\text { Bosnia and } \\
\text { Herzegovina } \\
\end{array}$ & Croatia & Slovenia & \begin{tabular}{|c|} 
Czech \\
Republick
\end{tabular} & Hungary & $\begin{array}{l}\text { United } \\
\text { Kingdom } \\
\end{array}$ & Australia \\
\hline title & + & + & + & + & + & + & + & + & + & + \\
\hline ISBN & & & + & & & & + & + & + & \\
\hline editor(s) & & & + & & & & & & & \\
\hline publisher & & & + & & & & & & & + \\
\hline publishers' data: headquarter of the publisher & & & & & & & & & & + \\
\hline publication language & + & & + & + & & & & & & \\
\hline indexing of conference proceeding: in CPCI & & & & & + & & + & + & & \\
\hline indexing of conference proceeding: in SCOPUS & & & & & & & + & + & & \\
\hline $\begin{array}{l}\text { indexing of conference proceeding: in a predefined list } \\
\text { of databases }\end{array}$ & & & & & & & & + & & \\
\hline $\begin{array}{l}\text { editorial committee structure: total number editorial } \\
\text { committee members }\end{array}$ & & & + & & & & & & & \\
\hline $\begin{array}{l}\text { editorial committee structure: total number of } \\
\text { countries from which the committee members } \\
\text { originate }\end{array}$ & & & + & & & & & & & \\
\hline
\end{tabular}

Table 3

Evaluation metadata for conference publication

\begin{tabular}{|c|c|c|c|c|c|c|c|c|c|c|}
\hline & Serbia & Macedonia & Montenegro & $\begin{array}{c}\text { Bosnia and } \\
\text { Herzegovina }\end{array}$ & Croatia & Slovenia & $\begin{array}{c}\text { Czech } \\
\text { Republick }\end{array}$ & Hungary & $\begin{array}{c}\text { United } \\
\text { Kingdom }\end{array}$ & Australia \\
\hline title & + & + & + & + & + & + & + & + & + & + \\
\hline author(s) & + & + & + & + & + & + & + & + & + & + \\
\hline publication year & + & + & + & + & + & + & + & + & + & + \\
\hline total number of pages & + & + & + & + & + & + & + & + & + & + \\
\hline conference & + & + & + & + & + & & & + & & + \\
\hline conference proceeding & & & + & & + & & & + & & + \\
\hline DOI/URL & & & & & & & + & & + & + \\
\hline scientific area & & & & & & + & + & + & + & + \\
\hline abstract & & & & & & & & & + & \\
\hline type of evaluation entity & + & + & + & + & + & + & & & & \\
\hline citation data: total number of citations & & & & & & + & & + & + & \\
\hline citation data: number of pure citation & & & & & & & & + & & \\
\hline citation data: number of pure citation in the last 5 & & & & & & + & & & & \\
\hline citation data: number of pure citation in the last 10 & & & & & & + & & & & \\
\hline $\begin{array}{l}\text { citation data: number of pure citation not derived from } \\
\text { the authors own affiliation }\end{array}$ & & & & & & & & + & & \\
\hline $\begin{array}{l}\text { citation data: number of pure citation found in } \\
\text { predefined list of databases }\end{array}$ & & & & & & & & + & & \\
\hline
\end{tabular}

\section{Conference Evaluation-related Data in the CERIF Model}

CRIS is an information system that stores bibliographic and normative data for entities related to conference results, as well as data on their interrelations. Because the CERIF standard and its physical model are a basis of CRIS systems, a legitimate conclusion is that the CERIF model should be investigated against the existing support of the relevant evaluation data, as well as against the possible extensions if needed. 
With CERIF, it is possible to determine the following entities: people involved in research activities (e.g. authors, organization members, etc.), organizations (e.g. universities, government agencies, publishing houses, etc.), research projects, research results (scientific publications, patents, etc.), etc. There are 8 main groups of CERIF entities. Base entities represent the core (basic) model entities (cfPers, cfOrgUnit and cfProject). Result entities represent the results from scientific research (cfRestPubl, cfResProd and cfResPat). Infrastructure entities represent the infrastructure that is relevant for scientific research (like cfEquip, cfFacil etc.). $2^{\text {nd }}$ Level entities further describe the Base and Result Entities (e.g. cfEvent, etc.). Indicator and Measure entities are used to define the research impact and supporting claims of that impact, covering the abovementioned entities (cfIndic and cfMeas). Multiple language entities provide multilingualism for CERIF data, like cfResPublTitle. Semantic layer entities cfClass (classes) and cfClassScheme (classification schemes) enable a rich semantic representation of data. CERIF prescribes a vocabulary that might be utilized for establishing classification, e.g. class "Author" of scheme "Person Output Contributions" can be used to define the person that is the author of a conference paper. Link entities are used to state timedetermined relations among other entities, like relation of a person and a publication cfPers_ResPubl. Every Link entity is described with a role (cfClassId, cfClassShemeId), timeframe of relation (cfStartDate, cfEndDate), value (cfFraction) and identifiers of elements creating the relation (e.g. cfPersId, cfResPublId). The "role" in link entities is not stored directly as an attribute value, but as references to Semantic layer entities.

\subsection{The Data in the CERIF Model}

The CERIF model Version 1.5 is used in this paper as a basis for proposing a model for evaluation of the results from conferences. The existing CERIF model for storing proceedings, conference results and conference data entities is represented in Figure 1. For simplicity and readability of the diagram, the classification entity cfClass was omitted.

The data about proceedings and conference results in CERIF can be placed in entities cfResPubl, cfResPublTitle and cfResPublAbstr. The title of a paper is acquired from $c$ ResPublTitle, while the abstract of a proceeding paper is acquired from the entity cfResPublabstr. The type of publication is set up by classification of instances of cfResPubl via cfResPubl_Class. CERIF scheme Output Types and its classifications from the controlled vocabulary (e.g. Conference Proceedings, Conference Proceedings Article, Conference Poster, Conference Abstract, Conference Contribution, etc.) are used for that.

Conference data can be stored in the event entity cfEvent and its name cfEventName. The event is stated as a conference via the cfEvent_Class entity and by the CERIF scheme Event Types and class Conference. The links between instances of publications and events are saved in the entity cfResPubl_Event. 
The researchers that are authors, editors or reviewers of a publication can be represented by instances of entities cfPers, cfPersName and cfPersName_Pers, which store information about a person and persons' name. An instance of cfPers is connected to instance $c f R e s P u b l$ with the link entity cfPers_ResPubl.

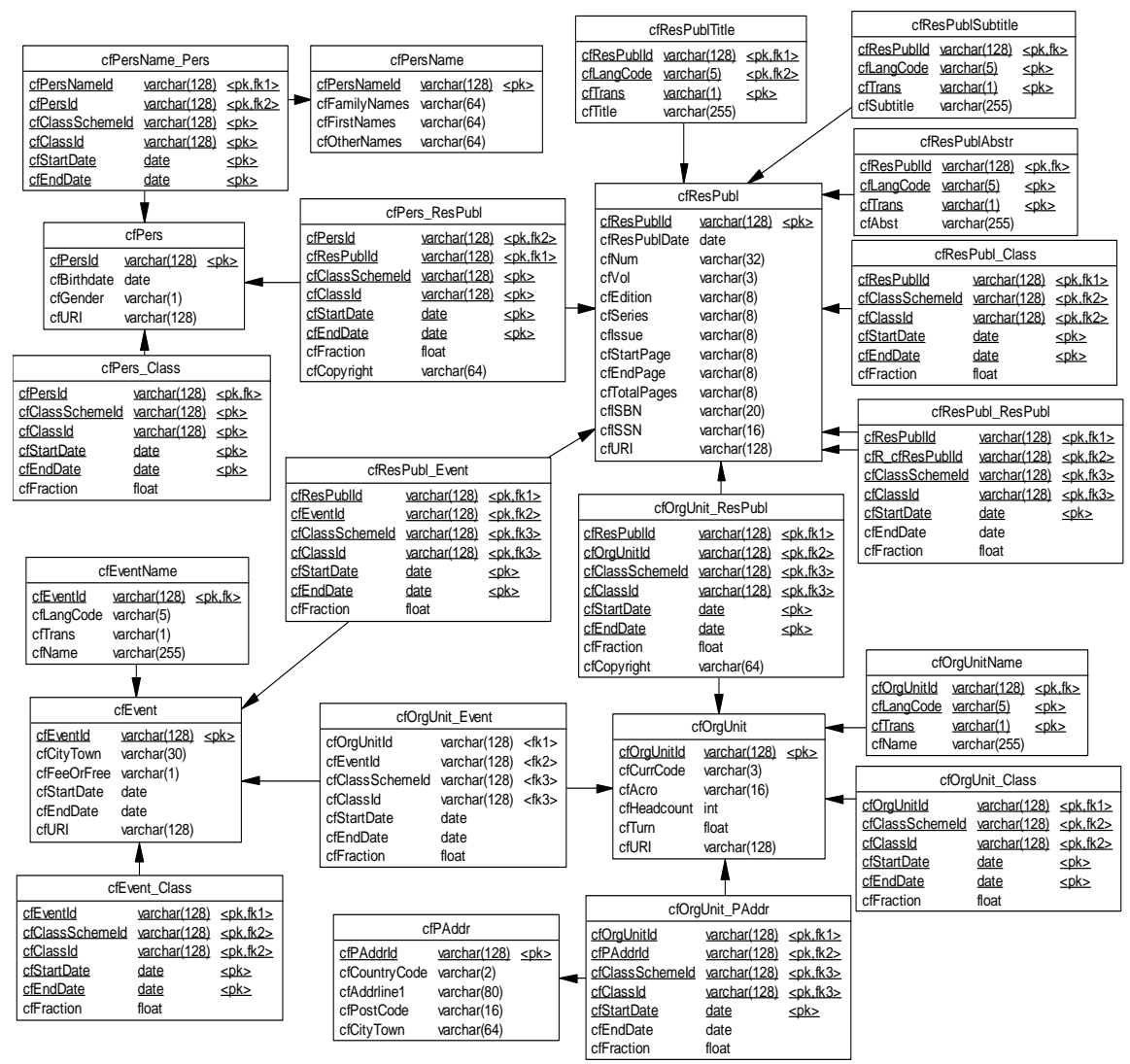

Figure 1

Existing CERIF entities for storing proceedings, conference results and conference data

The publisher of proceedings or the organizer of a conference can be represented by instances of entities cfOrgUnit (information about organizations) and cfOrgUnitName (organizations' names). The information about the headquarters (place) of an organization is acquired from the attributes cfCountryCode and cfCityTown of the entity cfPAdrr, which is connected to the organization through an instance of cfOrgUnit_PAdrr entity. Organizations are linked to publications and events with link entities cfOrgUnit_ResPubl and cfOrgUnit_Event respectively. A role of an organization that is a publisher (of proceeding) is enabled with CERIF scheme Organisation Output Roles and class Publisher. The role of a conference organizer is defined by using CERIF scheme Organisation Output Contributions and class Host. 


\subsection{CERIF Extension for Evaluation of Conference Publications}

CRIS UNS is a CERIF compatible research management system that has been developing since 2008 at the University of Novi Sad in the Republic of Serbia ${ }^{18}$. An extension to CERIF model which incorporates metadata for evaluation of journal articles in CRIS UNS was proposed in [20], while an extension to CERIF model aimed at modelling and storing a rulebook was proposed in [21]. The previously proposed CERIF model extension related to rulebook representation enables representation of rulebooks that relies on classifications.

An extension to CERIF aimed at providing the data necessary for evaluation in accordance with the findings from Section 3 is accomplished by relying on (using "as is" or repurposing) some of the existing CERIF entity attributes and by adding new semantics (classifications schemes and classes) for the existing CERIF entities. The semantics is defined to comply with the analyzed rulebooks. For the purpose of enabling a relation between a complex result and its constituents, a new class Belongs to and the corresponding scheme General Relations are added to CERIF vocabulary. The class Belongs to is used to classify entities cfResPubl_ResPubl (stating the inner relations among publications) and cfResPubl_Event (stating the relation between proceeding/paper and conference).

cfResPubl attributes (Figure 1) provide evaluation information for publication year (cfResPublDate), ISBN or ISMN identifier ( $c f I S B N)$, DOI or URL identifier $(c f U R I)$ and the total number of publication pages (cfTotalPages). The publication language is acquired from the entity cfResPublTitle. Assuming that the original language of the title is the same as the language of the publication, the attribute cfLangCode can be interpreted as publication language. The scientific area (group) for proceedings papers is provided via an instance of entity cfResPubl_Class, by stating the relation with the appropriate scientific area/group (the classification of scientific area/group is already defined within the rulebook). The indexing of a conference proceeding in CPCI or SCOPUS is enabled through instances of the entity cfResPubl_Class. The classification is enabled by a new scheme Proceeding Evaluation Details and three new classifications Is indexed in CPCI, Is indexed in SCOPUS and Is indexed in a predefined list of databases.

The year and place of a conference are obtained from cfEvent attributes containing data on timeframe (cfStartDate, cfEndDate), as well as the country and city (cfCountryCode, cfCityTown) of the conference. Assuming the same for cfEventName as for cfResPublTitle, the attribute cfLangCode of cfEventName can be utilized to acquire conference presentation language.

${ }^{18}$ http://www.cris.uns.ac.rs 
The organizer type for a conference is enabled through the entity cfOrgUnit_Class that is used for classifying organization (cfOrgUnit) as international/national organizer. The categorization of organizer is done by new scheme Organiser Evaluation Details and its classes International Organiser and National Organiser.

The data necessary for evaluation, which is measurable (e.g. total number of committee members, total number of papers, total number of citations etc.), can be stored by relying on CERIF Indicator and Measure entities (Figure 2). The authors of [17] used those entities to store metrics for comparison of universities (research inputs, process, outputs and outcomes). With cfIndic and cfMeas it is possible to create various quality and quantity indicators which can be related to the base, result and $2^{\text {nd }}$ level entities.

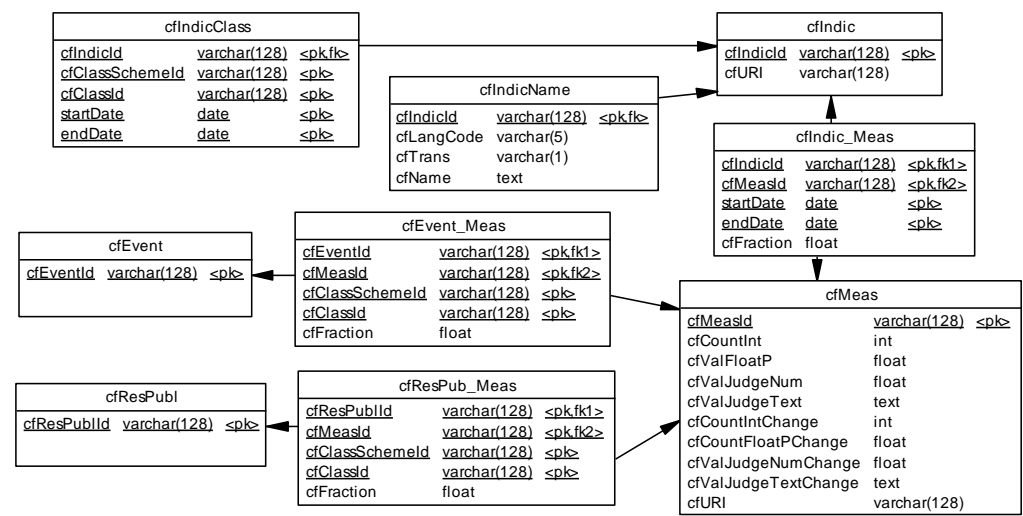

Figure 2

Storing measurements for publications and events

Every measurement identified in section 3 is represented as an instance of $c$ flndic (semantics of the measured value). The name of the measurement is kept in the multilingual entity cfIndicName. The classification of instances is done via the entity cfIndic_Class and a newly created scheme Evaluation Indicator Measurement and its classes. The class Citation data classifies the cfIndic instances Total number of citations, Number of pure citations, Number of pure citations in the last 5 years, Number of pure citations in the last 10 years, Number of pure citations not derived from the author's own affiliation and Number of pure citations found in a predefined list of databases. The classification Conference committee structure is used for cfIndic instances named Total number of committee members, Total number of countries from which committee members originate and Number of committee members from the most represented country. The class Conference results data is applied to cfIndic instances named Total number of papers, Number of submitted papers and Number of papers whose authors are foreigners. The class Editorial committee structure classifies the cfIndic instances Total number of committee members and Total number of 
countries from which the committee members originate. The concrete value of measurements for a single publication or a single event are defined as instances of cfMeas, storing integer (cfCountInt), float (cfValFloatP) or textual data (cfJudgeText) corresponding to the measured value. Measurements are connected by links (cfResPubl_Meas, cfEvent_Meas) to entities that are characterized by measurements (e.g. cfEvent_Meas connects an instance cfMeas storing Total number of committee members with a cfEvent instance representing a conference). The classification of those links is done with the class Belongs To from the scheme General Relations. The same class is used to link measurement and indicator (e.g. cfMeas is linked to cfIndic by the link entity cfIndic_Meas).

\section{Validation: The Case Study of the Serbian Rulebook}

In the Serbian rulebook, the Ministry prescribes the classification (categorization) of research results for Serbian researchers. The Serbian rulebook prescribes classification (categorization) of research results organizing them into hierarchical levels. Conferences can be categorized as international (M30) or national (M60) scientific meetings. So, a scientific result belonging to a M30 conference can be categorized as invited talk paper printed in full (M31), invited talk paper printed as abstract (M31), paper printed in full (M33), paper printed as abstract (M34), authorized discussion paper (M35), and editorial of proceedings (M36). The categorization of scientific results belonging to an M60 conference is similar to the one used for an M30 conference.

In order to provide a flexible and efficient mechanism for representation of machine-readable rulebooks aimed at automated evaluation of conference papers, we propose a solution relying on a rule-based expert system. For the purpose of representing rulebooks and evaluation rules, a Jess (Java Expert System Shell ${ }^{19}$ ) rule-based system is selected. Jess programming language is a Lisp-like declarative rule-based language that is very easy to read and understand by nonprogrammers, which was the main reason for our commitment to Jess as a solution to a rulebook representation task.

\subsection{Jess Implementation of the Serbian Rulebook}

The Serbian rulebook and its classification scheme are represented in Jess as facts. All fact templates are derived from Java object representation (Java Bean Classes) of the proposed CERIF extension. An example of a Jess code illustrating that

${ }^{19}$ http://herzberg.ca.sandia.gov/ 
principle can be "deftemplate CfMeas (declare (from-class CfMeas))", where a Jess shadow template CfMeas is created by looking at Java class representing CERIF CfMeas entity. The primitive Java class properties (e.g. cfCountInt of type integer) are mapped by default to Jess template slots with the same name and the same/similar type (e.g. Jess INTEGER). Non-primitive Java properties (if any) are mapped to Jess OBJECT type slots with the same name as corresponding Java properties. Since the slots of type OBJECT in Jess hold a reference to the Java object itself, the original objects from Java are always easily accessible.

The data for the facts representing the Serbian rulebook is extracted from Java object instances of extended CERIF model entities RuleBook, RuleBook_Class, RuleBookName, RuleBookDescr, RuleBook_ResearchersRole, RuleBook_ResultsType, RuleBook_EntityType, ResultsTypeMeasure, whose modelling is presented in [21].

Every evaluation rule form the Serbian rulebook can be formulated as a Jess rule with a distinct priority, where LHS (Left Hand Side) is composed of the fact statements that are all connected with logical conjunction by default. This priority enables avoidance of multiple classifications (e.g. higher priority is assigned to the rules classifying a result as "international" rather than the ones classifying it as "national"). All rules (Jess .clp files) for evaluation of conference results are available at http://s000.tinyupload.com/?file_id=95392329127505429716.

The proposed concept CERIF model extension for storing evaluation data for conference papers and the use of Jess rules language and reasoning engine for assigning categories to both conference and conference result (conference paper) are evaluated and verified by assessing the conference paper:

Nikolic, S., Penca, V., Ivanovic, D. (2014) "System for Modelling Rulebooks for the Evaluation of Scientific-Research Results. Case Study: Serbian Rulebook", Proceedings of the $4^{\text {th }}$ International Conference on Information Society and Technology (ICIST 2014) Society for Information Systems and Computer Networks, Kopaonik, Serbia, March 9-13, 2014, pp. 102-107

Following the evaluation process by the Serbian rulebook, the conference ICIST 2014 should first receive a category, before it is possible to categorize its papers. So, the higher priority rules for categorizing the conference are executed prior to the rules for categorizing papers. Also, in accordance with the Serbian rulebook, Jess will first try to classify the conference as international (M30) and, if that fails, it will attempt to apply other rules following the priority order (e.g. national conferences - M60, excluded from evaluation, etc.).

srRuleBook_international_conference (Figure 3) is a rule that classifies the conference as a M30 type.

The first paragraph in the rule checks if $4^{\text {th }}$ International Conference on Information Society and Technology (ICIST 2014) is a CERIF event (CfEvent with 
variable ?cfEventId) that is classified as a conference. ICIST 2014 (?cfEventId) has a class (cfOrgUnit_Class) with attribute cfClassId having the value "Conference", so the condition " $\{$ cfClassId $==$ Conference $\}$ " is met.

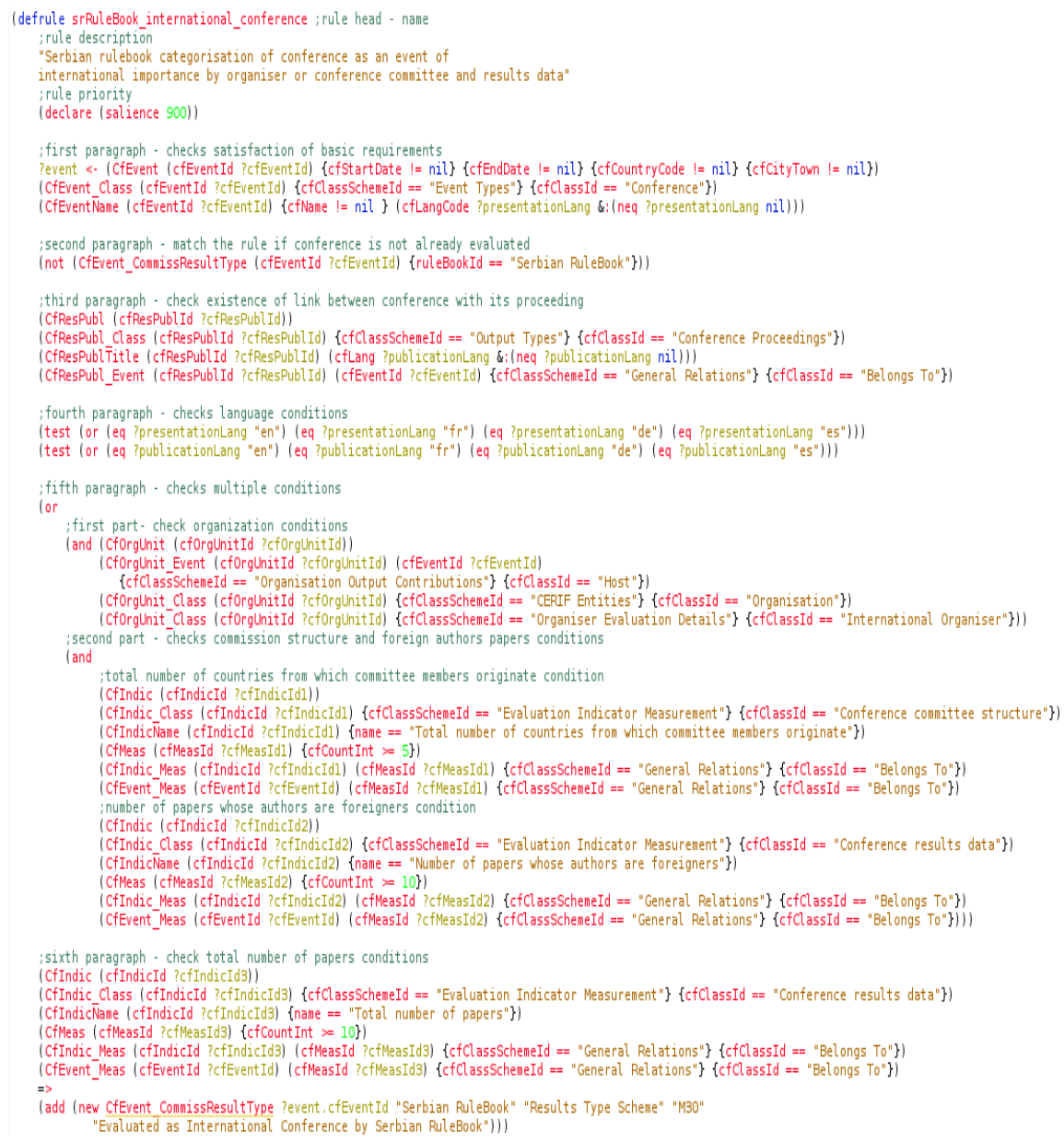

Figure 3

Rule for classifying CERIF events as M30 - conference of international importance

By the Serbian rulebook, an event must satisfy minimum requirements (e.g. data for name, place, year, etc. must be provided) to be considered for evaluation (first paragraph of the rule). ICIST 2014 was organized from 09/03/2013 (value stored in attribute cfStartDate) to 13/03/2013 (value stored in attribute cfEndDate) at Kopaonik (value stored in attribute cfCityTown), Serbia (value stored in attribute cfCountryCode), so the conditions "\{cfStartDate != nil\} \{cfEndDate != nil\} $\{$ ffCountryCode $!=$ nil $\}\{c f$ CityTown $!=$ nil $\} "$ are satisfied. The conference has a name (cfEventName) with attributes cfName and cfLangCode, where cfEventName 
stores the value "International Conference on Information Society and Technology (ICIST 2014)" and cfLangCode stores the value "en" (conference presentation language). The variable ?presentationLang holds the value that is acquired from cfLangCode. The attribute cfName and variable ?presentationLang fulfil the conditions "\{cfName != nil\}" and "(neq ?presentationLang nil)". This means that all required data for a conference categorization is provided.

For an event to be an international conference, its presentation and proceeding publication languages must be worldwide accepted. The third paragraph checks if there is a connection between the conference and its proceedings. ICIST 2014 (?cfEventId) has proceedings Proceedings of the $4^{\text {th }}$ International Conference on Information Society and Technology (?cfResPublId) whose title (cfResPublName) attribute cfLangCode stores value "en" (conference publication language). The variable ?publicationLang holds the value that is acquired from cfLangCode. The condition "(neq ?presentationLang nil)" is met. The fourth paragraph checks the abovementioned language conditions. At ICIST 2014 the papers were presented and published in the English language, so language conditions "(eq ?presentationLang "en")" and "(eq ?publicationLang "en")" are fulfilled.

According to Serbian rulebook, international conferences are those whose organizer is an international scientific association/institution or whose committee and results have international characteristics (committee members are from at least 5 countries, the conference must have at least 10 papers whose authors are foreigners). That claim is defined by the fifth paragraph, where two logical statements (conjunctions) are connected via $O R$ operator. The first conjunction statement checks the conditions for organization (CfOrgUnit). For ICIST 2014 (?cfEventId) there is an organizer Information Society of Serbia (?cfOrgUnitId) that is classified as "National Organiser" (value of attribute cfClassId of class cfOrgUnit_Class). So, the organizer condition "(cfClassId $==$ "International Organiser")" is not met. The second conjunction checks if the conditions (values of measures) related to international characteristics of conference committee structure and conference results are met. The measures are defined as two individual indicators (CfIndic), each having a concrete measurement (CfMeas) that is linked to the event. Since ICIST 2014 conference committee members were from 17 countries, Total number of countries from which the committee members originate (?cfIndicIdl) has a measurement ?cfMeasIdl with an attribute cfCountInt having the value 17 , so the condition " $\{c f$ CountInt $>=5\}$ " is satisfied. There were 29 papers whose authors are from foreign countries, so Number of papers whose authors are foreigners (?cfIndicId2) has a measurement ?cfMeasId 2 with an attribute $c f$ CountInt that has a value of 29 , which means that the condition " $\{$ cfCountInt $>=10\}$ " is met.

By the rulebook, the conference is excluded from evaluation if the total number of accepted papers is less than 10 (the sixth paragraph). Following the same principle as for the other explained measures, 85 papers from ICIST 2014 satisfy the last condition Total number of papers. 
Having fulfilled the conditions publication language, presentation language, conference committee structure and conference results data, the conference is categorized as M30.

Once a conference receives a category, the conference paper "System for modelling rulebooks for the evaluation of scientific-research results. Case study: Serbian Rulebook" is assessed by the conference paper rules. The rule srRuleBook_paper_printed_inFull_or_asAbstract for categorizing M30 conference papers (Conference Proceedings Article or Conference Poster) is applied. That rule relies on the value total number of pages (attribute cfTotalpages) of CERIF publication entity CfResPubl, to determine the paper category (paper published in full - M33 or paper published as abstract - M34). That value is 6 , so the paper is categorized as M33.

After the example mentioned above, we can conclude that by utilizing a rulebased expert system, it is feasible to evaluate research results in Serbia. By analogy, all other research results from the Serbian rulebook can be evaluated.

\section{Conclusion}

This paper investigates the current developments for evaluation of conference results in a broader research information environment that include various research evaluation data, with the primary aim to propose a data model and tools for automated evaluation of papers presented at scientific conferences.

In the first step, we have carried out and presented an analysis of selected national rulebooks regarding conference results evaluation. Then we proposed an extension to the CERIF model that supports evaluations in line with the results of the analysis. For evaluation of conference results, we have confirmed that all evaluation data required by the analyzed rulebooks are provided by the proposed CERIF model extension. By utilizing that extension, which can be applied to any CERIF like CRIS system, the evaluations on institution or national level should be easily introduced to research information systems.

For the automation of the evaluation process we propose an approach that utilizes the Jess rule language for representing a rulebook and its evaluation rules, and Jess inference engine for automated evaluation. The proposed approach was verified through an example of the Serbian rulebook and evaluation of a paper published at an international conference. Following that same principle, it can be shown that evaluation for other rulebooks and commissions can be accomplished by the same approach. This result could be useful for evaluation commissions, hence only a facts and rules should be specified, while all the evaluation is done by the inference engine.

The application of the proposed approach has certain constraints, regarding both the proposed CERIF extension and automation, of the evaluation process. Regarding the CERIF extension/model, the constraint is its support, since it analyses only current versions of the selected rulebooks. This, together with future 
developments related to scientific results evaluation, could require reassessment and revision of the proposed CERIF extension/model in the future. Regarding the automation of the evaluation process, the constraint is that writing extensive and complex Jess rules could require the involvement of engineering experts. This could be resolved by applying new developments related to knowledge representation and reasoning over such representations.

Both constraints will be the main activity of our future work.

\section{Acknowledgement}

This work was supported by the Ministry of Science and Technological Development of the Republic of Serbia, through project no.III-47003.

\section{References}

[1] A. Reinhardt and European Science Foundation, Evaluation in Research and Research Funding Organisations: European Practices: a Report, Strasbourg, European Science Foundation, 2012, ISBN: 9782918428831

[2] Committee for the Evaluation of Research - CIVR, "Guidelines for Research Evaluation", Ministry of University and Research (MIUR) 2006, from: http://vtr2006.cineca.it/documenti/linee_guida_EN.pdf

[3] M. Y. Vardi, "Revisiting the Publication Culture in Computing Research", Communications of the ACM, Vol. 53, No. 3, pp. 5-5, Mar. 2010

[4] J. Russell and R. Rousseau, Bibliometrics and Institutional Evaluation, Encyclopedia of Life Support Systems (EOLSS) Part 19.3 Science and Technology Policy. Oxford, Eolss Publishers, 2002

[5] J. Bar-Ilan, "Informetrics at the Beginning of the $21^{\text {st }}$ Century-A Review", Journal of Informetrics, Vol. 2, No. 1, pp. 1-52, Jan. 2008

[6] F. Moksony, R. Hegedűs, and M. Császár, "Rankings, Research Styles, and Publication Cultures: a Study of American Sociology Departments", Scientometrics, Vol. 101, No. 3, pp. 1715-1729, Dec. 2014

[7] D. Surla, D. Ivanović, Z. Konjović, and M. Racković, "Rules for Evaluation of Scientific Results Published in Scientific Journals", Management Information Systems, Vol. 7, No. 3, pp. 003-010, 2012

[8] R. Lister and I. Box, "A Citation Analysis of the ACE2005 - 2007 proceedings, with reference to the June 2007 CORE conference and journal rankings," in Tenth Australasian Computing Education Conference (ACE 2008), Wollongong, NSW, Australia, 2008, Vol. 78, pp. 93-102

[9] P. Küngas, S. Karus, S. Vakulenko, M. Dumas, C. Parra, and F. Casati, "Reverse-Engineering Conference Rankings: What does It Take to Make a Reputable Conference?”, Scientometrics, Vol. 96, No. 2, pp. 651-665, Aug. 2013 
[10] K. Jeffery, N. Houssos, B. Jörg, and A. Asserson, "Research Information Management: the CERIF Approach", International Journal of Metadata, Semantics and Ontologies, Vol. 9, No. 1, pp. 5-14, 2014

[11] V. Penca, S. Nikolic, D. Ivanovic, Z. Konjovic, and D. Surla, "SRU/Wbased CRIS Systems Search Profile", Program: Electronic Library and Information Systems, vol. 48, No. 2, pp. 140-166, 2014, DOI: 10.1108/PROG-07-2012-0040

[12] T. Seljak and A. Bošnjak, "Researchers' Bibliographies in COBISS.SI", Information Services and Use, Vol. 26, No. 4, pp. 303-308, Jan. 2006

[13] S. Nikolić, V. Penca, D. Ivanović, D. Surla, and Z. Konjović, "CRIS Service for Journals and Journal Articles Evaluation", in Proceedings of the $11^{\text {th }}$ International Conference on Current Research Information Systems, Prague, Czech Republic, 2012, pp. 323-332

[14] A. Reinhardt and European Science Foundation, Evaluation in Research and Research Funding Organisations: European Practices: a Report. Strasbourg: European Science Foundation, 2012

[15] S. Nikolić, V. Penca, and D. Ivanović, "Storing of Bibliometric Indicators in CERIF Data Model," presented at the ICIST 2013 - International Conference on Internet Society Technology, Kopaonik mountain resort, Republic of Serbia, 2013

[16] D. Ivanović, D. Surla, and M. Racković, “A CERIF Data Model Extension for Evaluation and Quantitative Expression of Scientific Research Results", Scientometrics, Vol. 86, No. 1, pp. 155-172, 2011

[17] A. Clements, B. Jörg, G. C. Lingjærde, T. Chudlarský, and L. Colledge, "The Application of the CERIF Data Format to Snowball Metrics", Procedia Computer Science, Vol. 33, pp. 297-300, 2014

[18] D. A. Waterman, "How do Expert Systems Differ from Conventional Programs?”, Expert Systems, Vol. 3, No. 1, pp. 16-19, Jan. 1986

[19] J. W. Lloyd, "Practical Advantages of Declarative Programming", in Joint Conference on Declarative Programming, GULP-PRODE, 1994

[20] D. Ivanovic, D. Surla, and M. Rackovic, "Journal Evaluation Based on Bibliometric Indicators and the CERIF Data Model", Computer Science and Information Systems, Vol. 9, No. 2, pp. 791-811, 2012

[21] S. Nikolic, V. Penca, and D. Ivanovic, "System for Modelling Rulebooks for the Evaluation of Scientific-Research Results. Case Study: Serbian Rulebook", in Proceedings of the $4^{\text {th }}$ International Conference on Information Society and Technology (ICIST 2014) Kopaonik, Serbia, 2014, pp. 102-107 